\title{
Notes About a New Metric on the Cotangent Bundle
}

\author{
Filiz Ocak*
}

(Communicated by Arif Salimov)

\begin{abstract}
In this article, we construct a new metric $\breve{G}={ }^{R} \nabla+\sum_{i, j=1}^{m} a^{j i} \delta p_{j} \delta p_{i}$ in the cotangent bundle, where ${ }^{R} \nabla$ is the Riemannian extension and $a^{j i}$ is a symmetric $(2,0)$-tensor field on a differentiable manifold.
\end{abstract}

Keywords: Cotangent bundle; Riemannian extension; geodesic; para-Nordenian metric.

AMS Subject Classification (2010): Primary: 53C07; Secondary: $53 C 15$.

\section{Introduction}

Cotangent bundle of differentiable manifold with a Riemannian extension, which was introduced by Patterson and Walker [11], was investigated by many authors [2,3,4,7,12,17]. Riemannian extension has been developed in several ways. Calviño-Louzao et.al [5] introduced the modified Riemannian extension using a symmetric tensor field of type $(0,2)$ and studied some geometric applications. Gezer and his collaborators studied the curvature properties and the Kähler- Norden structure with respect to the modified Riemannian extension [8]. Aslanci and Cakan [1] discussed the curvature properties of the deformed Riemannian extension in the cotangent bundle by means of musical isomorphism between tangent and cotangent bundle. Then Salimov and Cakan [13] investigated the deformed Riemannian extension using twin Norden metric.

In this paper, after the introduction and preliminaries, in section 3, we construct a new metric on the cotangent bundle using the Riemannian extension and quadratic differential form $\sum_{i, j=1}^{m} a^{j i} \delta p_{j} \delta p_{i}$, where $\delta p_{j}=$ $d p_{j}-p_{h} \Gamma_{i j}^{h} d x^{j}$. Then we calculate Levi-Civita connection and components of the curvature tensor for this metric. In section 4 , we get the necessary condition for the horizontal lift of any connection on the cotangent bundle to be a metric connection. In section 5, we investigate the geodesics on the cotangent bundle with respect to the new metric. Then we obtain the horizontal lift of a geodesic on $(M, g)$ that does not need to be a geodesic on $\left(T^{*} M, \breve{G}\right)$. In section 6 , we investigate the almost para-Nordenian, the para- Kählerian and the para-Nordenian properties of the new metric in the cotangent bundle.

\section{Preliminaries}

Let $M$ be an m-dimensional $C^{\infty}$-manifold with torsion-free connection $\nabla, T^{*} M$ be the cotangent bundle of $M$ and $\pi: T^{*} M \rightarrow M$ be the natural projection. For any local coordinates $\left(U, x^{i}\right), i=1, \ldots, m$ on $M$, we denote by $\left(\pi^{-1}(U), x^{i}, x^{\bar{i}}=p_{i}\right), \bar{i}=m+1, \ldots, 2 m$ the corresponding local coordinates on $T^{*} M$, where $x^{\bar{i}}=p_{i}$ are the components of the covector $p$ in each cotangent space $T_{x}^{*} M, x \in U$ with respect to the natural coframe $\left\{d x^{i}\right\}$. Let $F(M)\left(F\left(T^{*} M\right)\right)$ be the ring of real-valued $C^{\infty}$ functions on $M\left(T^{*} M\right)$ and $\Im_{s}^{r}(M)\left(\Im_{s}^{r}\left(T^{*} M\right)\right)$ be the module over $F(M)\left(F\left(T^{*} M\right)\right)$ of $C^{\infty}$ tensor fields of type $(\mathrm{r}, \mathrm{s})$.

The local expression of a vector and covector field is given by $Z=Z^{i} \frac{\partial}{\partial x^{i}}$ and $\theta=\theta_{i} d x^{i}$ in $U \subset M$, respectively. With respect to the natural frame $\left\{\frac{\partial}{\partial x^{i}}, \frac{\partial}{\partial x^{i}}\right\}$, then the vertical lift ${ }^{V} \theta \in \Im_{0}^{1}\left(T^{*} M\right)$ of $\theta \in \Im_{1}^{0}(M)$, the horizontal 
and complete lifts ${ }^{H} Z,{ }^{C} Z \in \Im_{0}^{1}\left(T^{*} M\right)$ of $Z \in \Im_{0}^{1}(M)$ are given by

$$
\begin{aligned}
& { }^{V} \theta=\sum_{i} \theta_{i} \frac{\partial}{\partial x^{\bar{i}}}, \\
& { }^{H} Z=Z^{i} \frac{\partial}{\partial x^{i}}+\sum_{i} p_{h} \Gamma_{i j}^{h} Z^{j} \frac{\partial}{\partial x^{\bar{i}}}, \\
& { }^{C} Z=Z^{i} \frac{\partial}{\partial x^{i}}-\sum_{i} p_{h} \partial_{i} Z^{h} \frac{\partial}{\partial x^{\bar{i}}},
\end{aligned}
$$

where the coefficients $\Gamma_{i j}^{h}$ are the Christoffel symbols of the Levi-Civita connection $\nabla$ on $M$ (for details, see [17]).

In [17], the adapted frame $\left\{\tilde{e}_{(\alpha)}\right\}=\left\{\tilde{e}_{(j)}, \tilde{e}_{(\bar{j})}\right\}$ is given by

$$
\begin{aligned}
& \tilde{e}_{(j)}={ }^{H} Z_{(j)}=\frac{\partial}{\partial x^{j}}+\sum_{h} p_{a} \Gamma_{h j}^{a} \frac{\partial}{\partial x^{\bar{h}}}, \\
& \tilde{e}_{(\bar{j})}={ }^{V} \omega^{(j)}=\frac{\partial}{\partial x^{\bar{j}}} .
\end{aligned}
$$

From (2.1), (2.2), (2.3) and (2.4), in the the adapted frame $\left\{\tilde{e}_{(\alpha)}\right\}$, we see that ${ }^{V} \theta,{ }^{H} Z$ and ${ }^{C} Z$ have the following components

$$
\begin{gathered}
{ }^{V} \theta=\sum_{i} \theta_{i} \tilde{e}_{(\bar{i})}, \quad{ }^{V} \theta=\left({ }^{V} \theta^{\alpha}\right)=\left(\begin{array}{c}
0 \\
\theta_{i}
\end{array}\right) \\
{ }^{H} Z=Z^{i} \tilde{e}_{(i)}, \quad{ }^{H} Z=\left({ }^{H} Z^{\alpha}\right)=\left(\begin{array}{c}
Z^{i} \\
0
\end{array}\right), \\
{ }^{C} Z=Z^{i} \tilde{e}_{(i)}-p_{h} \nabla_{i} Z^{h} \tilde{e}_{(\bar{i})}, \quad{ }^{C} Z=\left({ }^{C} Z^{\alpha}\right)=\left(\begin{array}{c}
Z^{i} \\
-p_{h} \nabla_{i} Z^{h}
\end{array}\right) .
\end{gathered}
$$

By (2.4), we consider local 1-forms $\tilde{\eta}^{\alpha}$ and vector field $\tilde{e}_{\beta}$ in $\pi^{-1}(U)$ given by

$$
\tilde{\eta}^{\alpha}=\overline{\mathrm{A}}^{\alpha}{ }_{B} d x^{B}, \tilde{e}_{\beta}=\mathrm{A}_{\beta}{ }^{A} \partial_{A}
$$

where

$$
A^{-1}=\left(\bar{A}_{B}^{\alpha}\right)=\left(\begin{array}{cc}
\bar{A}^{i}{ }_{j} & \bar{A}^{i}{ }_{\bar{j}} \\
\bar{A}^{i}{ }_{j} & \bar{A}^{i}{ }_{\bar{j}}
\end{array}\right)=\left(\begin{array}{ll}
\delta_{j}^{i} & 0 \\
-p_{a} \Gamma_{i j}^{a} & \delta_{i}^{j}
\end{array}\right)
$$

and

$$
A=\left(A_{\beta}{ }^{A}\right)=\left(\begin{array}{cc}
A_{j}{ }^{i} & A_{\bar{j}}{ }^{i} \\
A_{j}{ }^{i} & A_{\bar{j}}{ }^{i}
\end{array}\right)=\left(\begin{array}{ll}
\delta_{j}^{i} & 0 \\
p_{a} \Gamma_{i j}^{a} & \delta_{i}^{j}
\end{array}\right) .
$$

Also, the set $\left\{\tilde{\eta}^{\alpha}\right\}$ is the coframe dual to the adapted frame $\left\{\tilde{e}_{(\beta)}\right\}$, i.e. $\tilde{\eta}^{\alpha}\left(\tilde{e}_{(\beta)}\right)=\bar{A}^{\alpha}{ }_{B} A_{\beta}{ }^{B}=\delta_{\beta}^{\alpha}$.

The Lie bracket of the adapted frame $\left\{\tilde{e}_{(\alpha)}\right\}$ on $T^{*} M$ is given by

$$
\left[\tilde{e}_{\gamma}, \tilde{e}_{\beta}\right]=\Omega_{\gamma \beta}{ }^{\alpha} \tilde{e}_{\alpha}
$$

where

$$
\Omega_{\gamma \beta}{ }^{\alpha}=\left(\tilde{e}_{\gamma} A_{\beta}{ }^{A}-\tilde{e}_{\beta} A_{\gamma}{ }^{A}\right) \bar{A}^{\alpha}{ }_{A} .
$$

Using (2.4), (2.8) and (2.9), we have the non-zero components of $\Omega_{\gamma \beta}{ }^{\alpha}$ as follows

$$
\left\{\begin{array}{l}
\Omega_{l \bar{j}} \bar{i}_{\bar{i}}^{\bar{i}}=-\Omega_{\bar{j} l}^{\bar{i}}=-\Gamma_{l i}^{j} \\
\Omega_{l j}=p_{a} R_{l j i}{ }^{a}
\end{array}\right.
$$

where $R_{l j i}{ }^{a}$ is the local components of the curvature tensor $R$ of $\nabla$. 


\section{New metric $\breve{G}$ on $T^{*} M$}

The Riemannian extension ${ }^{R} \nabla \in \Im_{2}^{0}\left(T^{*} M\right)$ describes a pseudo-Riemannian metric in $T^{*} M$. The line element of the Riemannian extension ${ }^{R} \nabla$ is determined by

$$
d s^{2}=2 d x^{i} \delta p_{i},
$$

where $\delta p_{i}=d p_{i}-p_{h} \Gamma_{j i}^{h} d x^{i}$ (see [11, 17] for details).

Using the Riemannian extension and the quadratic differential form $\sum_{i, j=1}^{m} a^{j i} \delta p_{j} \delta p_{i}$, where $\delta p_{i}=d p_{i}-$ $p_{h} \Gamma_{j i}^{h} d x^{i}$ and $a^{j i}$ denote the components of a symmetric tensor field of type $(2,0)$ on $M$, we have a new metric

$$
\breve{G}=2 d x^{j} \delta p_{i}+\sum_{i, j=1}^{m} a^{j i} \delta p_{j} \delta p_{i}
$$

on $T^{*} M$ (for $a^{j i}=g^{i j}$, see [10]).

From (2.9) and (3.1), in the adapted frame $\left\{\tilde{e}_{(\alpha)}\right\}$, the metric $\breve{G}$ has the following components

$$
\breve{G}=\left(\begin{array}{cc}
\breve{G}_{j i} & \breve{G}_{j \bar{i}} \\
\breve{G}_{\bar{j} i} & \breve{G}_{\bar{j} \bar{i}}
\end{array}\right)=\left(\begin{array}{cc}
0 & \delta_{j}^{i} \\
\delta_{i}^{j} & a^{j i}
\end{array}\right) .
$$

By (2.1),(2.2) and (3.2), we have

$$
\begin{aligned}
& \breve{G}\left({ }^{H} V,{ }^{H} Z\right)=0, \\
& \breve{G}\left({ }^{H} Z,{ }^{V} \beta\right)={ }^{V}(\beta(Z))=\beta(Z) \circ \pi, \\
& \breve{G}\left({ }^{V} \omega,{ }^{V} \beta\right)={ }^{V}(\tilde{a}(\omega, \beta))=\tilde{a}(\omega, \beta) \circ \pi
\end{aligned}
$$

for any $V, Z \in \Im_{0}^{1}(M)$ and $\omega, \beta \in \Im_{1}^{0}(M)$, where $\tilde{a}$ is a symmetric tensor field of type $(2,0)$ on $M$. The $(0,2)$ tensor field on $T^{*} M$ is entirely detected by action on the vector fields of type ${ }^{H} Z$ and ${ }^{V} \beta$ (see [17, p.280]). So $\breve{G}$ is completely determined by the equation (3.3).

From (2.2) and (2.3), we see that the complete $\operatorname{lift}^{C} Z$ of $Z \in \Im_{0}^{1}(M)$ is expressed by

$$
{ }^{C} Z={ }^{H} Z-{ }^{V}(p(\nabla Z)),
$$

where $p(\nabla Z)=p_{k}\left(\nabla_{i} Z^{k}\right) d x^{i}$. Using (3.3) and (3.4), we get

$$
\tilde{G}\left({ }^{C} V,{ }^{C} Z\right)=-{ }^{V}[(p(\nabla V))(Z)+(p(\nabla Z))(V)+\tilde{a}(p(\nabla V), p(\nabla Z))],
$$

where $\tilde{a}(p(\nabla V), p(\nabla Z))=a^{i j}\left(p_{t} \nabla_{i} V^{t}\right)\left(p_{m} \nabla_{j} Z^{m}\right)$. Then we say that this metric is completely determined with vector fields of type ${ }^{C} V$ and ${ }^{C} Z$ on $T^{*} M$ (see [17, p.237]).

From (3.5), we obtain the following theorem:

Theorem 3.1. The complete lifts ${ }^{C} V,{ }^{C} Z$ of two vector fields $V, Z$ to $T^{*} M$ with metric $\breve{G}$ are orthogonal if $V, Z$ are parallel.

In [17, p.238 and p.277], we know that the Lie bracket for the horizontal, vertical and complete lifts of vector fields on the cotangent bundle $T^{*} M$ of $M$ satisfies the following:

$$
\begin{aligned}
& {\left[{ }^{H} V,{ }^{H} Z\right]={ }^{H}[V, Z]+\gamma R(V, Z)={ }^{H}[V, Z]+{ }^{V}(p R(V, Z)),} \\
& \left.{ }^{H} Z,{ }^{V} \theta\right]={ }^{V}\left(\nabla_{Z} \theta\right), \\
& \left.{ }^{V} \omega,{ }^{V} \theta\right]=0 \\
& \left.{ }^{C} V,{ }^{H} Z\right]={ }^{H}[V, Z]+{ }^{V}\left(p\left(L_{V} \nabla\right) Z\right), \\
& \left.{ }^{C} Z,{ }^{V} \theta\right]={ }^{V}\left(L_{Z} \theta\right)
\end{aligned}
$$

for any $V, Z \in \Im_{0}^{1}(M)$ and $\omega, \theta \in \Im_{1}^{0}(M)$. 
Theorem 3.2. Given an m-dimensional manifold $(M, g)$ and its cotangent bundle $\left(T^{*} M, \breve{G}\right)$. In the adapted frame $\left\{\tilde{e}_{(\alpha)}\right\}$, the Levi-Civita connection $\breve{\nabla}$ of the metric $\breve{G}$ satisfies the following equations:

$$
\begin{aligned}
& \text { i) } \breve{\nabla}_{\tilde{e}_{i}} \tilde{e}_{j}=\left(\Gamma_{i j}^{l}+\frac{1}{2} p_{s} R_{i j t}{ }^{s} a^{l t}\right) \tilde{e}_{l}+\left(p_{s} R_{l j i}{ }^{s}\right) \tilde{e}_{\bar{l}}, \\
& \text { ii) } \widetilde{\nabla}_{\tilde{e}_{i}} \tilde{e}_{\bar{j}}=\left(\frac{1}{2} \nabla_{i} a^{j l}-\Gamma_{i t}^{l} a^{l t}\right) \tilde{e}_{l}+\left(-\Gamma_{i l}^{j}+p_{s} R_{l i t}{ }^{s} a^{j t}\right) \tilde{e}_{\bar{l}}, \\
& \text { iii }) \widetilde{\nabla}_{\tilde{e}_{\bar{e}}} \tilde{e}_{j}=\left(\frac{1}{2} \nabla_{j} a^{l i}\right) \tilde{e}_{l}+\left(\frac{1}{2} p_{s} R_{l j t}{ }^{s} a^{i t}\right) \tilde{e}_{\bar{l}}, \\
& \text { iv) } \widetilde{\nabla}_{\tilde{e}_{\bar{i}}} \tilde{e}_{\bar{j}}=\left(-\frac{1}{2} \nabla_{l} a^{i j}\right) \tilde{e}_{\bar{l}},
\end{aligned}
$$

where $R_{l j i}{ }^{s}$ and $\Gamma_{i j}^{l}$ denote the components of the curvature tensor and coefficients of $\nabla$, respectively.

Proof. It is known that the Koszul formula for $\breve{\nabla}$ is given by

$$
\begin{aligned}
& 2 \breve{G}(\left.\breve{\nabla}_{Z} Y, V\right)=Z(\breve{G}(Y, V))+Y(\breve{G}(V, Z))-V(\breve{G}(Z, Y)) \\
&-\breve{G}(Z,[Y, V])+\breve{G}(Y,[V, Z])+\breve{G}(V,[Z, Y])
\end{aligned}
$$

for any $V, Y, Z \in \Im_{0}^{1}\left(T^{*} M\right)$. In the Koszul formula, we substitute $Z=\tilde{e}_{i}, \tilde{e}_{\bar{i}}, \quad Y=\tilde{e}_{j}, \tilde{e}_{\bar{j}}, \quad V=\tilde{e}_{k}, \tilde{e}_{\bar{k}}$. Using (2.10), (3.2) and the first Bianchi identity for the curvature tensor $R$, we do standard calculations.

Now we use $\breve{\nabla}_{e_{\alpha}} e_{\beta}=\breve{\Gamma}_{\alpha \beta}^{\delta} e_{\delta}$ with respect to the adapted frame $\left\{\tilde{e}_{(\alpha)}\right\}$ of $T^{*} M$, where the coefficients of the Levi-Civita connection $\breve{\nabla}$ of the metric $\breve{G}$ are denoted by $\breve{\Gamma}_{\alpha \beta}^{\delta}$. By using Theorem 3.2, we obtain

Corollary 3.1. Given an m-dimensional manifold $(M, g)$ and its cotangent bundle $\left(T^{*} M, \breve{G}\right)$. In the adapted frame $\left\{\tilde{e}_{(\alpha)}\right\}$, then the Christoffel symbols $\breve{\Gamma}_{\alpha \beta}^{\delta}$ of $\breve{\nabla}$ are found as follows:

$$
\begin{aligned}
\breve{\Gamma}_{i j}^{k} & =\Gamma_{i j}^{k}+\frac{1}{2} p_{s} R_{i j t}{ }^{s} a^{t k} & & \breve{\Gamma}_{i j}^{\bar{k}}=p_{s} R_{k j i}{ }^{s}, \\
\breve{\Gamma}_{i \bar{j}}^{k} & =\frac{1}{2} \nabla_{i} a^{j k}-\Gamma_{i t}^{j} a^{t k}, & & \bar{\Gamma}_{\bar{i} j}^{k}=\frac{1}{2} \nabla_{j} a^{i k}, \\
\breve{\Gamma}_{i \bar{j}}^{\bar{k}} & =-\Gamma_{i k}^{j}+\frac{1}{2} p_{s} R_{k i t}{ }^{s} a^{j t}, & & \bar{\Gamma}_{\bar{i} j}^{\bar{k}}=\frac{1}{2} p_{s} R_{k j t}{ }^{s} a^{t i}, \\
\breve{\Gamma}_{\bar{i} \bar{k}}^{\bar{k}} & =-\frac{1}{2} \nabla_{k} a^{i j}, & & \bar{\Gamma}_{\overline{i j}}^{k}=0 .
\end{aligned}
$$

Let $\hat{V}, \hat{Z} \in \Im_{0}^{1}\left(T^{*} M\right)$ and $\hat{V}=\hat{V}^{\alpha} \tilde{e}_{\alpha}, \quad \hat{Z}=\hat{Z}^{\beta} \tilde{e}_{\beta}$. In the adapted frame $\left\{\tilde{e}_{(\alpha)}\right\}$, the covariant derivative $\breve{\nabla}_{\hat{Z}} \hat{V}$ is given by

$$
\breve{\nabla}_{\hat{Z}} \hat{V}^{\alpha}=\hat{Z}^{\gamma} \tilde{e}_{\gamma} \hat{V}^{\alpha}+\breve{\Gamma}_{\gamma \beta}^{\alpha} \hat{V}^{\beta} \hat{Z}^{\gamma}
$$

Using (2.4), (2.5), (2.6), (3.8) and (3.9), we obtain

Proposition 3.1. Given an m-dimensional manifold $(M, g)$ and its cotangent bundle $\left(T^{*} M, \breve{G}\right)$. In the following, the Levi-Civita connection $\breve{\nabla}$ of the metic $\breve{G}$ provides

$$
\begin{aligned}
& i) \breve{\nabla}_{H} Z^{H} V={ }^{H}\left(\nabla_{Z} V\right)+\frac{1}{2}^{H}(\tilde{a} \circ p R(Z, V))+{ }^{V}(\tilde{V} R(Z, \tilde{p})), \\
& i i) \breve{\nabla}_{H} Z^{V} \omega=\frac{1}{2}{ }^{H}\left(\left(\nabla_{Z} \tilde{a}\right)(\omega,)\right)+{ }^{H}\left(\tilde{a} \circ \nabla_{Z} \omega\right)+{ }^{V}\left(\nabla_{Z} \omega\right)+\frac{1}{2} V(\tilde{a}(p R(, Z), \omega)), \\
& \quad i i i) \widetilde{\nabla}_{V \omega}{ }^{H} Z=\frac{1}{2}{ }^{H}\left(\left(\nabla_{Z} \tilde{a}\right)(\omega,)\right)+\frac{1}{2}^{V}(\tilde{Z} R(\tilde{\omega}, \tilde{p})), \\
& \quad i v) \bar{\nabla}_{V \omega}{ }^{V} \theta=-\frac{1}{2} V((\nabla \tilde{a})(\omega, \theta)) \\
& \text { for all } V, Z \in \Im_{0}^{1}(M), \omega, \theta \in \Im_{1}^{0}(M), \text { where }\left(\nabla_{Z} \tilde{a}\right)(\omega,)=\omega_{i} Z^{j} \nabla_{j} a^{l i}, \\
& V(\tilde{a}(p R(, Z), \omega))=a^{j t} p_{s} R_{l i t}{ }^{s} Z^{i} \omega_{j}, \tilde{Z}=g \circ Z \in \Im_{1}^{0}\left(M^{n}\right), \tilde{Z} R(Y, \tilde{p}) \in \Im_{1}^{0}\left(M^{n}\right) .
\end{aligned}
$$

\subsection{Curvature tensor of $\breve{\nabla}$}

Now, we investigate the curvature tensor $\breve{R}$ of $\left(T^{*} M, \breve{G}\right)$. We get

$$
\breve{R}\left(\tilde{e}_{(\alpha)}, \tilde{e}_{(\beta)}\right) \tilde{e}_{(\gamma)}=\breve{\nabla}_{\alpha} \breve{\nabla}_{\beta} \tilde{e}_{(\gamma)}-\breve{\nabla}_{\beta} \breve{\nabla}_{\alpha} \tilde{e}_{(\gamma)}-\Omega_{\alpha \beta}{ }^{\varepsilon} \breve{\nabla}_{\varepsilon} \tilde{e}_{(\gamma)},
$$




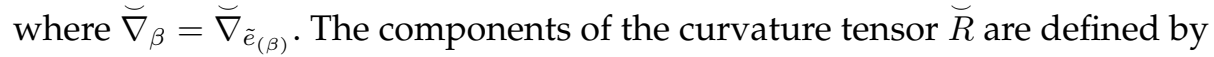

$$
\breve{R}_{\alpha \beta \gamma}{ }^{\sigma}=\tilde{e}_{\alpha} \breve{\Gamma}_{\beta \gamma}^{\sigma}-\tilde{e}_{\beta} \breve{\Gamma}_{\alpha \gamma}^{\sigma}+\breve{\Gamma}_{\alpha \varepsilon}^{\sigma} \breve{\Gamma}_{\beta \gamma}^{\varepsilon}-\breve{\Gamma}_{\beta \varepsilon}^{\sigma} \breve{\Gamma}_{\alpha \gamma}^{\varepsilon}-\Omega_{\alpha \beta}{ }^{\varepsilon} \breve{\Gamma}_{\varepsilon \gamma}^{\sigma}
$$

with respect to the adapted frame $\left\{\tilde{e}_{(\alpha)}\right\}$.

From (2.10) and (3.8), we find the components of $\breve{R}$ as follows:

$$
\begin{aligned}
& \breve{R}_{k i j}{ }^{l}=R_{k i j}{ }^{l}+\frac{1}{4} p_{s} p_{m} a^{n l} a^{f t}\left(R_{k t n}{ }^{s} R_{i j f}{ }^{m}-R_{i t n}{ }^{s} R_{k j f}{ }^{m}\right) \\
& +\frac{1}{2} p_{s} a^{l t}\left(\nabla_{k} R_{i j t}{ }^{s}-\nabla_{i} R_{k j t}{ }^{s}\right)-p_{s} a^{m l}\left(R_{t j k}{ }^{a} \Gamma_{i m}^{t}-R_{t j i}{ }^{a} \Gamma_{k m}^{t}\right) \\
& +\frac{1}{2} p_{s}\left(R_{i j t}{ }^{s} \nabla_{k} a^{t l}+R_{t k j}{ }^{s} \nabla_{i} a^{t l}+R_{t j i}{ }^{s} \nabla_{k} a^{t l}-R_{k i t}{ }^{s} \nabla_{j} a^{t l}\right) \text {, } \\
& \breve{R}_{\bar{k} i j}{ }^{l}=\frac{1}{2} R_{i j t}{ }^{k} a^{t l}-\frac{1}{2} \nabla_{i} \nabla_{j} a^{k l}+\frac{1}{2} p_{s} \Gamma_{i m}^{t} a^{m l} R_{t j f}{ }^{s} a^{f k} \\
& +\frac{1}{4} p_{s}\left(R_{i j m}{ }^{s} a^{m t} \nabla_{t} a^{k l}-R_{i t m}{ }^{s} a^{m l} \nabla_{j} a^{k t}-R_{t j f} a^{f k} \nabla_{i} a^{t l}\right), \\
& \breve{R}_{k i \bar{j}}^{l}=R_{i k t}{ }^{j} a^{t l}-\Gamma_{k t}^{j} \Gamma_{i f}^{t} a^{f l}+\Gamma_{i t}^{j} \Gamma_{k f}^{t} a^{f l}-\Gamma_{i t}^{j} \nabla_{k} a^{t l} \\
& +\Gamma_{i m}^{t} \nabla_{i} a^{t l}+\frac{1}{2}\left(\nabla_{k} \nabla_{i} a^{j l}-\nabla_{i} \nabla_{k} a^{j l}\right) \\
& +\frac{1}{4} p_{s}\left(R_{k t m}{ }^{s}\left(a^{m l} \nabla_{i} a^{j t}+a^{m j} \nabla_{i} a^{t l}\right)+R_{t i m}{ }^{s}\left(a^{m j} \nabla_{k} a^{t l}+a^{m l} \nabla_{k} a^{j t}\right)\right) \\
& +\frac{1}{2} p_{s}\left(R_{t k m}{ }^{s} a^{m l}\left(\Gamma_{i f}^{t} a^{f l}+\Gamma_{i f}^{j} a^{f t}\right)+R_{i t m}{ }^{s} a^{m l}\left(\Gamma_{k f}^{j} a^{f t}+\Gamma_{k f}^{t} a^{f l}\right)\right) \text {, } \\
& \breve{R}_{k i \bar{j}^{\bar{l}}}=R_{i k l}{ }^{j}+\frac{1}{4} p_{s} p_{m}\left(R_{l k f}{ }^{s} R_{t i n}{ }^{s} a^{f t} a^{n j}-R_{l i f}{ }^{s} R_{t k n}{ }^{m} a^{t f} a^{j n}\right) \\
& +\frac{1}{2} p_{s}\left(a^{m j} \nabla_{k} R_{l i m}{ }^{s}-a^{t j} \nabla_{i} R_{l k t}{ }^{s}\right)-p_{a} a^{m t}\left(R_{l t k}{ }^{a} \Gamma_{i m}^{j}-R_{l t i}{ }^{a} \Gamma_{k m}^{j}\right) \\
& +\frac{1}{2} p_{s}\left(R_{l i m}{ }^{s} \nabla_{k} a^{m j}-R_{l k m}{ }^{s} \nabla_{i} a^{m j}-R_{l t k}{ }^{s} \nabla_{i} a^{j t}\right) \\
& +\frac{1}{2} p_{s}\left(R_{k i t}{ }^{s} \nabla_{l} a^{t j}-R_{l t i}{ }^{s} \nabla_{k} a^{j t}\right), \\
& \breve{R}_{k i j}{ }^{\bar{l}}=p_{s}\left(\nabla_{k} R_{l j i}{ }^{s}-\nabla_{i} R_{l j k}{ }^{s}\right)+\frac{1}{2} p_{s} p_{m} a^{f t}\left(R_{l t k}{ }^{m} R_{i j f}{ }^{s}+R_{l k f}{ }^{m} R_{t j i}{ }^{s}\right) \\
& \text { - } \frac{1}{2} p_{s} p_{m} a^{f t}\left(R_{i t l}{ }^{m} R_{k j f}{ }^{s}+R_{l i f}{ }^{m} R_{t j k}{ }^{s}+R_{k i t}{ }^{m} R_{l j m}{ }^{s}\right) \text {, } \\
& \breve{R}_{\bar{k} i j}{ }^{\bar{l}}=R_{l j i}{ }^{k}-\frac{1}{2} p_{s} a^{t k} \nabla_{i} R_{l j t}{ }^{s} \\
& +\frac{1}{4} p_{s} p_{m}\left(R_{l t f}{ }^{s} R_{i j n}{ }^{m} a^{f k} a^{n t}-R_{l i f}{ }^{s} R_{t j n}{ }^{m} a^{n k} a^{f t}\right) \\
& \text { - } \frac{1}{2} p_{s}\left(R_{l j m}{ }^{s} \nabla_{i} a^{m k}+R_{t j i}{ }^{s} \nabla_{l} a^{k t}-R_{l t i}{ }^{s} \nabla_{j} a^{k t}\right) \text {, }
\end{aligned}
$$

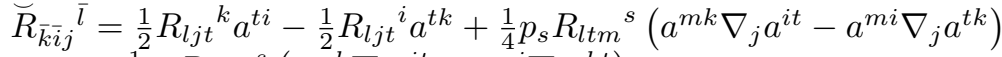

$$
\begin{aligned}
& +\frac{1}{4} p_{s} R_{t j m}{ }^{s}\left(a^{m k} \nabla_{l} a^{i t}-a^{m i} \nabla_{l} a^{k t}\right), \\
& \breve{R}_{\bar{k} i \bar{j}}{ }^{\bar{l}}=\frac{1}{2} R_{l i t}{ }^{k} a^{j t}+\frac{1}{2} \nabla_{i} \nabla_{l} a^{k j}-\frac{1}{2} p_{s} R_{l t m}{ }^{s} \Gamma_{i f}^{j} a^{m k} a^{f t} \\
& +\frac{1}{4} p_{s}\left(R_{l i f}{ }^{s} a^{f m} \nabla_{t} a^{k j}-R_{t i m} a^{j m} \nabla_{l} a^{k t}+R_{l t m}{ }^{s} a^{m k} \nabla_{i} a^{j t}\right), \\
& \breve{R}_{\bar{k} \bar{i} \bar{j}} \bar{l}=\frac{1}{4}\left(\nabla_{l} a^{k t} \nabla_{t} a^{i j}-\nabla_{l} a^{i t} \nabla_{t} a^{k j}\right), \\
& \breve{R}_{k \overline{i j}}^{l}=-\frac{1}{4}\left(\nabla_{k} a^{t l} \nabla_{t} a^{i j}+\nabla_{t} a^{i l} \nabla_{k} a^{j t}\right) \\
& +\frac{1}{2}\left(\Gamma_{k m}^{t} a^{m l} \nabla_{t} a^{i j}+\Gamma_{k m}^{j} a^{m t} \nabla_{t} a^{i l}\right), \\
& \breve{R}_{\bar{k} \bar{i} j}^{l}=\frac{1}{4}\left(\nabla_{t} a^{k l} \nabla_{j} a^{i t}-\nabla_{t} a^{l i} \nabla_{j} a^{k t}\right) \text {, } \\
& \breve{R}_{\bar{k} \bar{i} \bar{j}} l=0 \text {. }
\end{aligned}
$$

We have

Theorem 3.3. Given an m-dimensional manifold $(M, g)$ and its cotangent bundle $\left(T^{*} M, \breve{G}\right)$. Then $\left(T^{*} M, \breve{G}\right)$ is flat if $M$ is flat and $\nabla \tilde{a}=0$.

Proof. It immediately follows from last equations.

\section{The metric connection with respect to the metric $\breve{G}$}

We know that the metric connection satisfies $\bar{\nabla} \breve{G}=0$ and has non-trivial torsion tensor. By the definition of the horizontal lift ${ }^{H} \nabla$ of any connection $\nabla$ on $T^{*} M$, we write

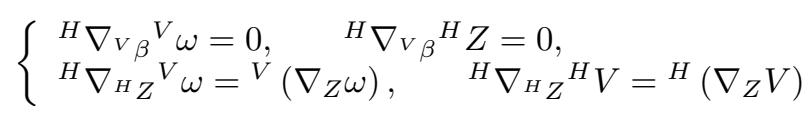

for any $V, Z \in \Im_{0}^{1}(M)$ and $\omega, \beta \in \Im_{1}^{0}(M)$. The torsion tensor $T$ of ${ }^{H} \nabla$ determined by

$$
\mathrm{T}\left({ }^{V} \omega,{ }^{V} \beta\right)=0, \quad \mathrm{~T}\left({ }^{H} Z,{ }^{V} \omega\right)=0, \quad \mathrm{~T}\left({ }^{H} V,{ }^{H} Z\right)=-\gamma R(V, Z),
$$


where $R$ denote the curvature tensor of $\nabla$ and $\gamma R(V, Z)=\sum_{i} p_{h} R_{k l i}{ }^{h} V^{k} Z^{l} \frac{\partial}{\partial x^{i}}[17, \mathrm{p} .287]$.

Using (3.3) and (4.1), we have

$$
\begin{aligned}
\left({ }^{H} \nabla_{H} Z\right. & \breve{G})\left({ }^{V} \beta,{ }^{V} \varepsilon\right)={ }^{H} \nabla_{H} Z \bar{G}\left({ }^{V} \beta,{ }^{V} \varepsilon\right)-\breve{G}\left({ }^{H} \nabla_{H}{ }^{V} \beta,{ }^{V} \varepsilon\right)-\breve{G}\left({ }^{V} \beta,{ }^{H} \nabla_{H}{ }^{V} \varepsilon\right) \\
& ={ }^{H} \nabla_{H}{ }^{V}(\tilde{a}(\beta, \varepsilon))-\breve{G}\left({ }^{V}\left(\nabla_{Z} \beta\right),{ }^{V} \varepsilon\right)-\breve{G}\left({ }^{V} \beta, V\left(\nabla_{Z} \varepsilon\right)\right) \\
& ={ }^{V}\left(\nabla_{Z}(\tilde{a}(\beta, \varepsilon))\right)-V\left(\tilde{a}\left(\nabla_{Z} \beta, \varepsilon\right)\right)-V\left(\tilde{a}\left(\beta, \nabla_{Z} \varepsilon\right)\right) \\
& ={ }^{V}(Z \tilde{a}(\beta, \varepsilon))-{ }^{V}\left(\tilde{a}\left(\nabla_{Z} \beta, \varepsilon\right)\right)-{ }^{V}\left(\tilde{a}\left(\beta, \nabla_{Z} \varepsilon\right)\right) \\
& ={ }^{V}\left(\left(\nabla_{Z} \tilde{a}\right)(\beta, \varepsilon)\right)
\end{aligned}
$$

and the others are zero. Then we have the following theorem:

Theorem 4.1. The horizontal lift ${ }^{H} \nabla$ of $\nabla$ is a metric connection of the metric $\breve{G}$ if and only if the symmetric $(2,0)$-tensor field $\tilde{a}$ on $(M, g)$ is parallel with respect to $\nabla$.

\section{Geodesics on $\left(T^{*} M, \breve{G}\right)$}

Now, let us investigate the geodesics of the $\left(T^{*} M, \breve{G}\right)$. Firstly, let $C: x^{h}=x^{h}(t)$ be a curve in $M$ and $\omega_{h}(t)$ be a covector field along $C$. We suppose that $\tilde{C}$ is a curve on $T^{*} M$ and locally given by

$$
x^{h}=x^{h}(t), x^{\bar{h}} \stackrel{\text { def }}{=} p_{h}=\omega_{h}(t) .
$$

The horizontal lift of the curve $C$ in $M$ satisfies the equation

$$
\frac{\delta \omega_{h}}{d t}=\frac{d \omega_{h}}{d t}-\Gamma_{j h}^{i} \frac{d x^{j}}{d t} \omega_{i}=0 .
$$

Hence, if the initial condition $\omega_{h}=\omega_{h}^{0}$ for $\omega_{h}=\omega_{h}^{0}$ is offered, then there exists a unique horizontal lift given by (5.1).

The differential equation of the geodesic in $\left(T^{*} M, \breve{G}\right)$ is expressed by the form

$$
\frac{\delta^{2} x^{A}}{d t^{2}}=\frac{d^{2} x^{A}}{d t^{2}}+\breve{\Gamma}_{C B}^{A} \frac{d x^{C}}{d t} \frac{d x^{B}}{d t}=0
$$

with respect to the induced coordinates $\left(x^{i}, x^{\bar{i}}\right)=\left(x^{i}, p_{i}\right)$ in $T^{*} M$, where $t$ is the arc length of a curve $x^{B}=x^{B}(t), B=(h, \bar{h})$ in $T^{*} M$ and $\breve{\Gamma}_{C B}^{A}$ are components of $\breve{\nabla}$ defined by (3.8).

By using the adapted frame $\left\{\tilde{e}_{(\alpha)}\right\}$, we can easily write the equation (5.2). Using (2.8), we get

$$
\eta^{\alpha}=\bar{A}^{\alpha}{ }_{A} d x^{A},
$$

i.e.

$$
\eta^{h}=\bar{A}_{A}^{h} d x^{A}=\delta_{i}^{h} d x^{i}=d x^{h}
$$

for $\alpha=h$ and

$$
\eta^{\bar{h}}=\bar{A}_{A}{ }_{A} d x^{A}=-p_{a} \Gamma_{h j}^{a} d x^{j}+\delta_{j}^{h} d x^{j}=\delta p_{h}
$$

for $\alpha=\bar{h}$. Also we put

$$
\begin{aligned}
& \frac{\eta^{h}}{d t}=\bar{A}^{h}{ }_{A} \frac{d x^{A}}{d t}=\frac{d x^{h}}{d t}, \\
& \frac{\eta^{h}}{d t}=\bar{A}_{A} \frac{d x^{A}}{d t}=\frac{\delta p_{h}}{d t}
\end{aligned}
$$

along a curve $x^{B}=x^{B}(t)$ in $T^{*} M$. So, we get the equation (5.2) which is equal to the following

$$
\frac{d}{d t}\left(\frac{\eta^{\alpha}}{d t}\right)+\breve{\Gamma}_{\gamma \beta}^{\alpha} \frac{\eta^{\gamma}}{d t} \frac{\eta^{\beta}}{d t}=0
$$

with respect to adapted frame $\left\{\tilde{e}_{(\alpha)}\right\}$. From (3.8), we obtain

$$
\begin{aligned}
& \text { a) } \frac{\delta^{2} x^{h}}{d t^{2}}+\frac{1}{2} p_{m} R_{i j t}{ }^{m} a^{t h} \frac{d x^{i}}{d t} \frac{d x^{j}}{d t}+\frac{1}{2} \nabla_{j} a^{i h} \frac{\delta p_{i}}{d t} \frac{d x^{j}}{d t}+\frac{1}{2}\left(\nabla_{i} a^{j h}-\Gamma_{i t}^{j} a^{t h}\right) \frac{d x^{i}}{d t} \frac{\delta p_{j}}{d t}=0, \\
& \text { b) } \frac{\delta^{2} p_{h}}{d t^{2}}+p_{m} R_{h j i}{ }^{m} \frac{d x^{i}}{d t} \frac{d x^{j}}{d t}+\frac{1}{2} p_{m} R_{h j t}{ }^{m} a^{i t} \frac{\delta p_{i}}{d t} \frac{d x^{j}}{d t}+p_{m} R_{h i t} a^{t j} \frac{d x^{i}}{d t} \frac{\delta p_{j}}{d t} \\
& \quad-\frac{1}{2} \nabla_{h} a^{i j} \frac{\delta p_{i} i}{d t} \frac{\delta p_{j}}{d t}=0 .
\end{aligned}
$$


Taking account of the local components of the curvature tensor $R$, i.e. $R_{i j k}{ }^{t}=\partial_{i} \Gamma_{j k}^{t}-\partial_{j} \Gamma_{i k}^{t}+\Gamma_{i m}^{t} \Gamma_{j k}^{m}-\Gamma_{j m}^{t} \Gamma_{i k}^{m}$ and antisymmetry with respect to $\mathrm{i}$ and $\mathrm{j}$, we find $R_{(i j) t}{ }^{m}=0$. Since $R_{(i j) t}{ }^{m}=0$, we have $R_{i j t}{ }^{m} \frac{d x^{i}}{d t} \frac{d x^{j}}{d t}=0$. So, we obtain

$$
\begin{aligned}
& \text { a) } \frac{\delta^{2} x^{h}}{d t^{2}}+\frac{1}{2} \nabla_{j} a^{i h} \frac{\delta p_{i}}{d t} \frac{d x^{j}}{d t}+\frac{1}{2}\left(\nabla_{i} a^{j h}-\Gamma_{i t}^{j} a^{t h}\right) \frac{d x^{i}}{d t} \frac{\delta p_{j}}{d t}=0 \\
& \text { b) } \frac{\delta^{2} p_{h}}{d t^{2}}+p_{m} R_{h j i} \frac{d x^{i}}{d t} \frac{d x^{j}}{d t}+\frac{1}{2} p_{m} R_{h j t}{ }^{m} a^{i t} \frac{\delta p_{i}}{d t} \frac{d x^{j}}{d t}+p_{m} R_{h i t} a^{t j} \frac{d x^{i}}{d t} \frac{\delta p_{j}}{d t} \\
& \quad-\frac{1}{2} \nabla_{h} a^{i j} \frac{\delta p_{i}}{d t} \frac{\delta p_{j}}{d t}=0 .
\end{aligned}
$$

Theorem 5.1. Let $\tilde{C}$ be a curve in $T^{*} M$ expressed locally by $x^{h}=x^{h}(t), p_{h}=\omega_{h}(t)$ with respect to the induced coordinates $\left(x^{i}, x^{\bar{i}}\right)=\left(x^{i}, p_{i}\right)$ in $T^{*} M$. The curve $\tilde{C}$ is a geodesic of $\breve{G}$, if it satisfies the equation (5.3).

Let now $\tilde{C}: x^{h}=x^{h}(t), x^{\bar{h}}=p_{h}(t)=\omega_{h}(t)$ be a horizontal lift $\left(\frac{\delta p_{h}}{d t}=\frac{\delta \omega_{h}}{d t}=\frac{d \omega_{h}}{d t}-\Gamma_{j h}^{i} \frac{d x^{j}}{d t} \omega_{i}=0\right)$ of the geodesic $C: x^{h}=x^{h}(t)\left(\frac{\delta^{2} x^{h}}{d t^{2}}=0\right)$ in $M$ of $\nabla$. Due to the nonvanishing second term of equation $(5.3, b)$, the geodesic equation (5.2) does not provide.

Theorem 5.2. The horizontal lift of a geodesic on $(M, g)$ needs not be a geodesic on $T^{*} M$ with respect to the connection $\vec{\nabla}$.

\section{Para-Nordenian structures on $\left(T^{*} M, \breve{G}\right)$}

An almost product structure $P \in \Im_{1}^{1}(M)$ is defined by $P^{2}=I$. Therefore, the pair $(M, g)$ is called an almost product manifold. An almost paracomplex manifold is an almost product manifold $(M, P), P^{2}=I$, such that the two eigenbundles $T^{+} M$ and $T^{-} M$ associated to the two eigenvalues +1 and -1 of $P$, respectively, have the same rank. We know that the dimension of an almost paracomplex manifold has to be even. Using the paracomplex structure $F$, we get the set $\{I, P\}$ on $M$, which is an isomorphic representation of the algebra of order 2, which is defined the algebra of paracomplex (or double) numbers and is given by $R(j), j^{2}=1$ [6].

If a tensor field $\vartheta \in \Im_{q}^{0}\left(M^{2 m}\right)$ satisfies

$$
\vartheta\left(P Z_{1}, Z_{2}, \ldots, Z_{q}\right)=\vartheta\left(Z_{1}, P Z_{2}, \ldots, Z_{q}\right)=\ldots=\vartheta\left(Z_{1}, Z_{2}, \ldots, P Z_{q}\right)
$$

for all $Z_{1}, Z_{2}, \ldots, Z_{q} \in \Im_{0}^{1}\left(M^{2 m}\right)$, then $\vartheta$ is called pure with respect to the paracomplex structure $P$.

By means of the paracomplex structure $P$ and the pure tensor field $\vartheta$, the operator $\Phi_{P}$ defined in [16] is

$$
\begin{gathered}
\left(\Phi_{P} \vartheta\right)\left(Y, Z_{1}, \ldots, Z_{q}\right)=(P Y)\left(\vartheta\left(Z_{1}, \ldots, Z_{q}\right)\right)-Y\left(\vartheta\left(P Z_{1}, Z_{2}, \ldots, Z_{q}\right)\right) \\
+\vartheta\left(\left(L_{Z_{1}} P\right) Y, Z_{2}, \ldots, Z_{q}\right)+\ldots+\vartheta\left(Z_{1}, Z_{2}, \ldots,\left(L_{Z_{q}} P\right) Y\right),
\end{gathered}
$$

where $L_{Y}$ is the Lie derivative with respect to $Y$ and $\Phi_{P} \vartheta \in \Im_{q+1}^{0}\left(M^{2 m}\right)$.

A tensor field $\vartheta$ is called an almost paraholomorphic with respect to the paracomplex algebra $R(j)$, if $\Phi_{P} \vartheta=0($ see $[9,15])$.

The pair $(P, g)$ is a para-Nordenian structure where $P$ is an almost paracomplex structure and $g$ is a pure tensor field with respect to $P$, i.e. $g(P V, Z)=g(V, P Z)$. Then a $2 \mathrm{~m}$-dimensional pseudo-Riemannian manifold $M$ with an almost para-Nordenian structure is called to be an almost para-Nordenian manifold. Furthermore, the almost para-Nordenian manifold is para-Kähler $\left(\nabla_{g} P=0\right)$ if and only if $g$ is paraholomorphic $\left(\Phi_{P} g=0\right)$ (see [14, 15]).

Given the cotangent bundle $T^{*} M$ with the metric $\breve{G}$. A tensor field $P \in \Im_{1}^{1}\left(T^{*} M\right)$ is expressed by

$$
\left\{\begin{array}{l}
P^{H} Z=-{ }^{H} Z \\
P^{V} \theta=-{ }^{V} \theta
\end{array}\right.
$$

for any $Z \in \Im_{0}^{1}(M)$ and $\theta \in \Im_{1}^{0}(M)$. By virtue of (6.1), we have

$$
\begin{gathered}
P^{2}\left({ }^{H} Z\right)=P\left(P^{H} Z\right)=P\left(-{ }^{H} Z\right)={ }^{H} Z \\
P^{2}\left({ }^{V} \theta\right)=P\left(P^{V} \theta\right)=P\left(-{ }^{V} \theta\right)={ }^{V} \theta
\end{gathered}
$$

for any $Z \in \Im_{0}^{1}(M)$ and $\theta \in \Im_{1}^{0}(M)$, i.e. $P^{2}=I$.

Theorem 6.1. The triple $\left(T^{*} M, \breve{G}, P\right)$ is an almost para-Nordenian manifold. 
Proof. Using purity condition

$$
W(V, Z)=\breve{G}(P V, Z)-\breve{G}(V, P Z)
$$

for any $V, Z \in \Im_{0}^{1}\left(T^{*} M\right)$, from (3.3) and (6.1) we have

$$
\begin{aligned}
& W\left({ }^{V} \omega,{ }^{V} \theta\right)=\breve{G}\left(P^{V} \omega,{ }^{V} \theta\right)-\breve{G}\left({ }^{V} \omega, P^{V} \theta\right)=0, \\
& W\left({ }^{H} Z,{ }^{V} \theta\right)=\breve{G}\left(P^{H} Z,{ }^{V} \theta\right)-\breve{G}\left({ }^{H} Z, P^{V} \theta\right)=-\breve{G}\left({ }^{H} Z,{ }^{V} \theta\right)+\breve{G}\left({ }^{H} Z,{ }^{V} \theta\right)=0, \\
& W\left({ }^{V} \theta,{ }^{H} Z\right)=-W\left({ }^{H} Z,{ }^{V} \theta\right)=0, \\
& W\left({ }^{H} V,{ }^{H} Z\right)=\breve{G}\left(P^{H} V,{ }^{H} Z\right)-\breve{G}\left({ }^{H} V, P^{H} Z\right)=-\breve{G}\left({ }^{H} V,{ }^{H} Z\right)+\breve{G}\left({ }^{H} V,{ }^{H} Z\right)=0
\end{aligned}
$$

i.e. $\breve{G}$ is pure with respect to $P$ as defined by (6.1). Hence, Theorem 6.1 is proved.

In view of Proposition 3.1 and (6.1), the covariant derivative of $P$ with respect to the metric $\breve{G}$ is

$$
\begin{aligned}
& \left(\breve{\nabla}_{H_{V}} P\right)\left({ }^{V} \theta\right)=\breve{\nabla}_{H_{V}}\left(P^{V} \theta\right)-P\left(\breve{\nabla}_{H_{V}}{ }^{V} \theta\right)=-\breve{\nabla}_{H} V\left({ }^{V} \theta\right)-P\left(\breve{\nabla}_{H_{V}}{ }^{V} \theta\right) \\
& =-\frac{1}{2}{ }^{H}\left(\left(\nabla_{V} \tilde{a}\right)(\omega,)\right)-{ }^{H}\left(\tilde{a} \circ \nabla_{V} \omega\right)-{ }^{V}\left(\nabla_{V} \omega\right)-\frac{1}{2}^{V}(\tilde{a}(p R(, V), \omega)) \\
& -\left(-\frac{1}{2}{ }^{H}\left(\left(\nabla_{V} \tilde{a}\right)(\omega,)\right)-{ }^{H}\left(\tilde{a} \circ \nabla_{V} \omega\right)-{ }^{V}\left(\nabla_{Y} \omega\right)-{ }^{\frac{1}{2}} V(\tilde{a}(p R(, V), \omega))\right)=0 \text {, } \\
& \left(\breve{\nabla}_{H}{ } P\right)\left({ }^{H} Z\right)=\breve{\nabla}_{H V}\left(P^{H} Z\right)-P\left(\breve{\nabla}_{H}{ }^{H} Z\right)=-\breve{\nabla}_{H}{ }^{H} Z-P\left(\breve{\nabla}_{H}{ }^{H} Z\right) \\
& =-\left({ }^{H}\left(\nabla_{V} Z\right)+\frac{1}{2}{ }^{H}(\tilde{a} \circ p R(V, Z))+{ }^{V}(\tilde{Z} R(V, \tilde{p}))\right) \\
& -\left(-{ }^{H}\left(\nabla_{V} Z\right)-\frac{1}{2}^{H}(\tilde{a} \circ p R(V, Z))-{ }^{V}(\tilde{Z} R(V, \tilde{p}))\right)=0, \\
& \left(\breve{\nabla}_{V \omega} P\right)\left({ }^{H} Z\right)=\breve{\nabla}_{V_{\omega}}\left(P^{H} Z\right)-P\left(\breve{\nabla}_{V_{\omega}}{ }^{H} Z\right)=\breve{\nabla}_{V_{\omega}}\left(-{ }^{H} Z\right)-P\left(\breve{\nabla}_{V_{\omega}}{ }^{H} Z\right) \\
& =-\frac{1}{2}^{H}\left(\left(\nabla_{Z} \tilde{a}\right)(\omega,)\right)-\frac{1}{2}^{V}(\tilde{Z} R(\tilde{\omega}, \tilde{p}))+\frac{1}{2}^{H}\left(\left(\nabla_{Z} \tilde{a}\right)(\omega,)\right)+\frac{1}{2}^{V}(\tilde{Z} R(\tilde{\omega}, \tilde{p}))=0 \text {, } \\
& \left(\breve{\nabla}_{V \omega} P\right)\left({ }^{V} \theta\right)=\breve{\nabla}_{V_{\omega}}\left(P^{V} \theta\right)-P\left(\breve{\nabla}_{V_{\omega}}{ }^{V} \theta\right)=\frac{1}{2} V((\nabla \tilde{a})(\omega, \theta)) \\
& -\frac{1}{2} V((\nabla \tilde{a})(\omega, \theta))=0 .
\end{aligned}
$$

Theorem 6.2. The triple $\left(T^{*} M, \breve{G}, P\right)$ is a para-Kählerian manifold.

The Nijenhuis tensor is given by the formula

$$
N_{P}(V, Z)=[P V, P Z]-P[P V, Z]-P[V, P Z]+P^{2}[V, Z]
$$

and the vanishing of the Nijenhuis tensor is characterized integrability of the almost paracomplex structure. If $P$ is integrable, we say that the almost para-Nordenian manifold is a para-Nordenian manifold (see [14]).

Using (3.6), (6.1) and (6.2), we find

$$
N_{P}\left({ }^{H} V,{ }^{H} Z\right)=N_{P}\left({ }^{H} V,{ }^{V} \theta\right)=N_{P}\left({ }^{V} \omega,{ }^{H} Z\right)=N_{P}\left({ }^{V} \omega,{ }^{V} \theta\right)=0
$$

i.e. $\left(T^{*} M, \breve{G}, P\right)$ is integrable. Then we have the following theorem:

Theorem 6.3. The triple $\left(T^{*} M, \breve{G}, P\right)$ is a para-Nordenian manifold.

If the Lie derivative of a vector field $\tilde{Z} \in \Im_{0}^{1}\left(T^{*} M\right)$ satisfies the condition $L_{\tilde{Z}} P=0$, then the vector field is said to be an almost paraholomorphic (see [9]).

Taking account of (3.6) and (6.1), we have

$$
\begin{aligned}
& \left(L_{C}{ }_{Z} P\right)^{V} \theta=L_{C} P^{V} \theta-P\left(L_{C}{ }^{V} \theta\right) \\
& =-L_{C}{ }^{V} \theta-P\left({ }^{V}\left(L_{Z} \theta\right)\right)=-{ }^{V}\left(L_{Z} \theta\right)+\left({ }^{V}\left(L_{Z} \theta\right)\right)=0, \\
& \left(L_{C} Z P\right){ }^{H} V=L_{C} P^{H} V-P\left(L_{C}{ }^{H} V\right)=-L_{C}{ }^{H} V-P\left(L_{C}{ }^{H} V\right) \\
& =-\left({ }^{H}[Z, V]+{ }^{V}\left(p\left(L_{Z} \nabla\right) Y\right)\right)-P\left({ }^{H}[Z, V]+{ }^{V}\left(p\left(L_{Z} \nabla\right) Y\right)\right)=0, \\
& \left(L_{V}{ } P\right)^{V} \theta=L_{V} \omega P^{V} \theta-P\left(L_{V}{ }^{V} \theta\right)=0 \\
& \left(L_{V} \omega\right){ }^{H} Z=L_{V}{ }^{H} P^{H} Z-P\left(L_{V_{\omega}}{ }^{H} Z\right)=-L_{V_{\omega}}{ }^{H} Z-P\left(L_{V_{\omega}}{ }^{H} Z\right) \\
& ={ }^{V}\left(\nabla_{Z} \omega\right)-{ }^{V}\left(\nabla_{Z} \omega\right)=0 \\
& \left(L_{H}{ }_{Z} P\right)^{V} \theta=L_{H} Z P^{V} \theta-P\left(L_{H}{ }^{V} \theta\right)=-\left[{ }^{H} Z,{ }^{V} \omega\right]-P\left(\left[{ }^{H} Z,{ }^{V} \omega\right]\right) \\
& =-{ }^{V}\left(\nabla_{Z} \omega\right)-P\left({ }^{V}\left(\nabla_{Z} \omega\right)\right)=0, \\
& \left(L_{H} Z P\right){ }^{H} V=L_{H} P^{H} V-P\left(L_{H} Z{ }^{H} V\right)=-\left[{ }^{H} Z,{ }^{H} V\right]-P\left(\left[{ }^{H} Z,{ }^{H} V\right]\right) \\
& =-\left({ }^{H}[Z, V]+{ }^{V}(p R(Z, V))\right)-P\left({ }^{H}[Z, V]+{ }^{V}(p R(Z, V))\right)=0 .
\end{aligned}
$$

Hence, we can conclude the following theorem: 
Theorem 6.4. The complete and horizontal lifts ${ }^{C} Z,{ }^{H} Z \in \Im_{0}^{1}\left(T^{*} M\right)$ of $Z \in \Im_{0}^{1}(M)$ and the vertical lift $V_{\omega} \in$ $\Im_{0}^{1}\left(T^{*} M\right)$ of $\omega \in \Im_{1}^{0}(M)$ are the almost paraholomorphic vector fields with respect to the almost para-Nordenian structure $(P, \breve{G})$.

\section{References}

[1] Aslanci, S., Cakan, R., On a cotangent bundle with deformed Riemannian extension, Mediterr. J. Math. 11 (2014), 1251-1260.

[2] Aslanci, S., Kazimova, S., Salimov, A.A., Some Remarks Concerning Riemannian Extensions, Ukrainian. Math. J. 62, (2010), 661-675.

[3] Bejan, C.L., Eken, Ş., A characterization of the Riemann extension in terms of harmonicity, Czech. Math. J. 67, (2017), 197-206.

[4] Bejan, C.L., Meriç, Ş. E., Kılıç, E., Einstein Metrics Induced by Natural Riemann Extensions, Adv. Appl. Clifford Algebras. 27, (2017), 23332343.

[5] Calviño-Louzao, E., García-Río, E., Gilkey, P., Vázquez-Lorenzo A., The Geometry of Modified Riemannian Extensions, Proc. R. Soc. Lond. Ser. A Math. Phys. Eng. Sci. 465, (2009), 2023-2040.

[6] Cruceanu, V., Fortuny, P., Gadea, M., A survey on paracomplex Geometry, Rocky Mountain J. Math. 26, (1995), 83-115.

[7] Dryuma, V., The Riemann Extensions in Theory of Differential Equations and their Applications, Mat. Fiz. Anal. Geom. 10, (2003), 307-325.

[8] Gezer, A., Bilen, L., Cakmak, A., Properties of Modified Riemannian Extensions, Zh. Mat. Fiz. Anal. Geom. 11, (2015), 159-173.

[9] Kruckovic, GI., Hypercomplex structures on manifolds I. Trudy. Sem. Vektor Tenzor Anal. 16, (1972), 174-201(in Russian).

[10] Ocak, F., Kazimova, S., On a new metric in the cotangent bundle, Transactions of NAS of Azerbaijan Series of Physical-Technical and Mathematical Sciences. 38, (2018), 128-138.

[11] Patterson, E.M., Walker, A.G., Riemann Extensions, Quart. J. Math. Oxford Ser. 3, (1952), 19-28.

[12] Salimov, A., Tensor Operators and Their Applications, Nova Science Publishers, New York, USA, 2012.

[13] Salimov, A., Cakan, R., On deformed Riemannian extensions associated with twin Norden metrics, Chinese Annals of Mathematics Series B. $36,(2015), 345-354$.

[14] Salimov, A.A., Iscan, M., Akbulut, K., Notes on para-Norden-Walker 4-manifolds, International Journal of Geometric Methods in Modern Physics. 7, (2010), 1331-1347.

[15] Salimov, A. A., Iscan, M. and Etayo, F., Paraholomorphic B-manifold and its properties, Topology Appl. 154,(2007), 925-933.

[16] Yano, K., Ako, M., On certain operators associated with tensor fields, Kodai Math. Sem. Rep. 20, (1968), 414-436.

[17] Yano, K. and Ishihara, S., Tangent and Cotangent Bundles, Pure and Applied Mathematics, 16, Marcel Dekker, Inc., New York, 1973.

\section{Affiliations}

\section{FILIZ OCAK}

AdDress: Karadeniz Technical University, Faculty of Sciences, Dept. of Mathematics, Trabzon, Turkey. E-MAIL: filiz_math@hotmail.com, filiz.ocak@ktu.edu.tr http:/ / orcid.org/0000-0003-4157-6404 